INPLASY

PROTOCOL

To cite: Zeng et al. Metaanalysis of the efficacy of hemoperfusion combined with hemodialysis in treating acute severe organophosphorus pesticide poisoning. Inplasy protocol 2021110056. doi: 10.37766/inplasy2021.11.0056

Received: 16 November 2021

Published: 16 November 2021

Corresponding author:

Siyao Zeng

357893553@qq.com

Author Affiliation:

Ningxia Medical University

Support: Ningxia Medical University.

Review Stage at time of this submission: Data analysis.

Conflicts of interest:

None declared.

\section{Meta-analysis of the efficacy of hemoperfusion combined with hemodialysis in treating acute severe organophosphorus pesticide poisoning}

Zeng, SY1; Ma, L2; Hu, XD3; Wang, C4; Gou, Y5; Li, Y6.

Review question / Objective: Our meta-analysis aims to conduct a more comprehensive and in-depth analysis of the efficacy of hemoperfusion combined with hemodialysis on ASOPP patients through several different outcome indicators, providing clinical evidence for ASOPP blood purification therapy.

Information sources: The PubMed, EMbase, Cochrane, Web of sciense, Ovid, Scopus, Chinese National Knowledge Infrastructure (CNKI), Chinese Biomedical literature (CBM), WanFang, Weipu (VIP), Chinese clinical trial Registry and Clinical Trials.gov databases.

INPLASY registration number: This protocol was registered with the International Platform of Registered Systematic Review and Meta-Analysis Protocols (INPLASY) on 16 November 2021 and was last updated on 16 November 2021 (registration number INPLASY2021110056).

\section{INTRODUCTION}

Review question / Objective: Our metaanalysis aims to conduct a more comprehensive and in-depth analysis of the efficacy of hemoperfusion combined with hemodialysis on ASOPP patients through several different outcome indicators, providing clinical evidence for ASOPP blood purification therapy.

Condition being studied: Lack of evidencebased medical evidence for hemoperfusion combined with hemodialysis in treating acute severe organophosphorus pesticide poisoning. 


\section{METHODS}

Participant or population: Acute severe organophosphorus pesticide poisoning.

Intervention: Hemoperfusion combined with hemodialysis.

Comparator: Conventional emergency treatment.

\section{Study designs to be included: RCT.}

Eligibility criteria: Articles were included if they met all of the following criteria:1) Randomized controlled study (RCT) 2) Study population consisted of accidental or suicidal OP poisoning patients, and diagnosed as acute severe organophosphorus pesticide poisoning (ASOPP) and there are clinical manifestations of pulmonary edema, respiratory failure, coma, cerebral edema or other important organ failures. 3) Study population consisted of patients who potentially received hemoperfusion combined with hemodialysis on the basis of conventional emergency treatment once they presented to the emergency department. 4) One or more of the following were reported:cure rate, efficacy, incidence of complications, time for cholinesterase level to return to normal, coma time, time of hospitalization and atropine dosage. 5) Articles were from January 1, 2017 to November 11, 2021.Articles were excluded if any of the following were present: 1)The article did not clearly state that the research was RCT.2) Studies were not published in Chinese or English. 3) Study data was unavailable or the content of article was not rigorous.4) Studies involving primarily special populations (e.g. AIDS or tuberculosis patients)5)Repeated literature6)Sample size was too small.

Information sources: The PubMed, EMbase, Cochrane, Web of sciense, Ovid, Scopus, Chinese National Knowledge Infrastructure (CNKI), Chinese Biomedical literature (CBM), WanFang, Weipu (VIP), Chinese clinical trial Registry and Clinical Trials.gov databases.
Main outcome(s): Cure rate, efficacy and incidence of complications.

Additional outcome(s): Time for cholinesterase level to return to normal, coma time, time of hospitalization and atropine dosage.

\section{Data management: NoteExpress.}

Quality assessment / Risk of bias analysis: Cochrane collaboration tool.

Strategy of data synthesis: We used the RevMan software (version 5.3) provided by the Cochrane Collaboration and Stata (version 14 and 16) for data analysis. Dichotomous variable was presented as Risk ratios (RR) and ordinal categorical variable was presented as Odds ratios (OR). Continuous outcomes were presented as the mean difference and with a $95 \%$ confidential interval $(\mathrm{Cl})$ rate.

Subgroup analysis: According to the publication year of the article, the proportion of men, the sample size, the average age and the unit of measurement for subgroup analysis.

Sensitivity analysis: After deleting any one of the documents, merge them again. If the effect size is not much different, then pass the sensitivity analysis.

\section{Language: English and Chinese.}

Country(ies) involved: China.

Keywords: hemoperfusion combined with hemodialysis, acute severe organophosphorus pesticide poisoning.

\section{Contributions of each author:}

Author 1 - Siyao Zeng - document retrieval, literature screening, data extraction, quality assessment statistical analysis and writing.

Email: 357893553@qq.com

Author 2 - Lei Ma - statistical analysis.

Email: 13895306161@163.com

Author 3 - Xiaodong $\mathrm{Hu}$ - quality assessment.

Email: 443535136@qq.com 
Author 4 - Cheng Wang - literature screening.

Email: 1761691737@qq.com

Author 5 - Yi Gou - data extraction and

verification.

Email: 1621878341@qq.com

Author 6 - Yi Li - document retrieval.

Email: 1966269327@qq.com 